\title{
Non-English speaking background patients in a predominantly English-speaking region may be more likely to present with a dementia other than Alzheimer's disease
}

\author{
Kevin Ong $^{1^{*}}$, Michael Woodward $^{2}$ \\ ${ }^{1}$ Department of Nuclear Medicine and Centre for PET, Austin Health, Heidelberg, Australia; \\ *Corresponding Author: drkevinong@gmail.com \\ ${ }^{2}$ Aged and Residential Care Services, Austin Health, Heidelberg West, Victoria, Australia
}

Received 22 April 2013; revised 22 May 2013; accepted 4 June 2013

Copyright (c) 2013 Kevin Ong, Michael Woodward. This is an open access article distributed under the Creative Commons Attribution License, which permits unrestricted use, distribution, and reproduction in any medium, provided the original work is properly cited.

\begin{abstract}
Information on 54 patients was retrospectively collected to compare the presentation trends of cognitive disorders in those of non-English speaking background (NESB) to English speaking background (ESB) attending an Australian memory clinic that extensively uses fluorodeoxyglucose positron emission tomography (FDG $P E T$ ) in the diagnosis of cognitive concerns. NESB patients were less likely to be diagnosed with Alzheimer's disease (AD) as the sole neurodegenerative diagnosis (Fisher exact test, $p=$ 0.08 ), and NESB patients with dementia were more likely to have non-AD dementia (Fisher exact test, $p=0.06$ ). They experienced symptoms 18 months longer before receiving a formal diagnosis $(\mathrm{t}(46)=2.2, \mathrm{p}=0.03)$. Older elderly NESB females were under represented in those presenting to the clinic (Fisher exact test, $p=$ 0.04). The clinical work-up of NESB patients as opposed to those of ESB relied more heavily on FDG PET (Fisher exact test, $p=0.04$ ). ESB and NESB patients may have different attitudes towards dementia, affecting how they present, and biomarkers may be more heavily relied on when language affects history taking and neuropsychological testing.
\end{abstract}

Keywords: Dementia; Memory Clinic; Non-English Speaking Background; Positron Emission

Tomography

\section{INTRODUCTION}

Memory clinics are increasingly being established as centres of diagnostic excellence [1]. One study that focused on equity of access to a memory clinic service serving the north-western suburbs of Melbourne Australia noted that non-English speaking background (NESB) patients were under represented [2]. Furthermore, NESB patients were more likely to be diagnosed with a psychiatric disorder, and present in the later stages of dementia, though they present with similar rates of dementia subtypes, compared to English speaking background (ESB) patients.

Rapid progress in the last few years in the dementia field includes translational studies promoting the integration into routine clinical practice of biomarkers like fluorodeoxyglucose positron emission tomography (FDG PET), which has been consistently demonstrated to have far greater sensitivity and specificity in discriminating between neurodegenerative subtypes, compared to clinical diagnostic criteria [3]. FDG PET also has the greatest contribution to routine tests for predicting mild cognitive impairment (MCI) conversion to Alzheimer's disease (AD) compared to cerebral spinal fluid protein levels or MR imaging [4]. Hence memory clinics that incorporate FDG PET as part of the diagnostic work-up may further improve diagnostic accuracy.

The aim of this study was to explore presentation and diagnostic trends of NESB and English speaking background (ESB) patients attending an Australian memory clinic service in Melbourne that extensively uses FDG PET in the diagnostic work-up process, and to see if these trends match those previously reported [2]. 


\section{METHODS}

\subsection{Participants and the Memory Service}

We conducted retrospective analyses of all attending patients' records whose initial consultation and/or feedback session at the Austin cognitive dementia and memory service (AMS) fell in the months of January 2010 and February 2010. Other patients who attended followup for other reasons during this period were not studied. The AMS is located in the north-eastern suburbs of Melbourne Australia and is widely regarded as a centre of excellence, providing assessments of predominantly local residents but also those from further away who pose diagnostic difficulties. Unlike other memory clinics [2], there is onsite access to FDG PET. In addition, one of the clinical staff is both a neurologist with a special interest in dementia as well as a nuclear medicine specialist with a special interest in PET neuroimaging. There is also a psychogeriatrician on staff.

\subsection{The Client Pathway}

The client pathway through the AMS is similar to that described previously [2]. However, instead of using the Cambridge Examination for Mental Disorders in the Elderly [5], initial cognitive examination of those sufficiently proficient in English is mainly performed using the Addenbrooke's Cognitive Examination Revised (ACER) [6] as well as the Geriatric Depression Scale [7]. The mini mental state examination (MMSE) [8] and clock drawing test scores are available from the ACER. MMSEs in some foreign languages including Greek and Italian are used via an interpreter if patients cannot communicate in English. An interpreter is also used for the overall assessment if the patient is unable to communicate sufficiently proficiently in English.

Structural neuroimaging with a CT and/or an MRI scan is also often performed to assess focal atrophy including hippocampal atrophy, assess any ischaemic changes, and exclude other causes of cognitive impairment. Where a firm diagnosis is not apparent during the initial consultation, because of the complexity of the presenting signs and symptoms and/or a language barrier, the patient is usually referred for a brain FDG PET scan, and/or a thorough neuropsychology assessment.

A case conference attended by all the clinical staff occurs shortly after all the investigations are completed, to decide on a formal diagnosis and the management plan to be given to the patient and carer, which are relayed at a subsequent feedback session. The clinical staff are familiar with the cultural and language barriers which the NESB patients face, and this is given careful consideration when deciding on a formal diagnosis. Hence diagnoses are not rigidly based on psychometry test performance, which is carefully interpreted in the context of a patient's circumstances. Diagnoses are based on standard criteria, for example Petersen's criteria [9] for MCI, NIAReagan criteria for AD, NINDS-AIREN criteria for vascular dementia (VD), Lund-Manchester criteria for Frontotemporal dementia (FTD), and the International Consensus Consortium criteria for dementia with Lewy bodies (DLB).

This study was approved by the Austin Health Human Research Ethics Committee.

\subsection{Statistical Analysis}

Categorically-scaled data were analysed using Fisher's exact test. Continuously-scaled data were analysed using Student's t-test. Statistical significance was deemed to have been attained when the two-tailed p-value was less than 0.05. Data was reported as mean (SD) unless otherwise indicated.

\section{RESULTS}

\subsection{Patient Baseline Characteristics}

Of the 110 appointments booked in January and February 2010, 31 were initial consultations, and 23 were feedback sessions. The mean age of patients was 73.5 years, 50\% were female, and 35\% were of NESB (Table 1) (The country of origin for NESB patients is given in Supplementary Table 1). A trend towards greater proportion of ESB patients $>75$ years of age compared to NESB patients was noted, and this was driven by the significantly lower proportion of female NESB patients $>75$ year of age compared to female ESB patients (Fisher exact test, $p=0.04$ ). While the reported rates of ESB and NESB patients living with their spouses/partners were similar, NESB patients were less likely to live alone (Fisher exact test, $\mathrm{p}=0.08$ ). NESB patients were also less likely to have received at least secondary education (Fisher exact test, $p<0.001$ ), and performed worse on cognitive testing. For example, they scored 3.3 points on average lower in the MMSE $(\mathrm{t}(51)=2.57, \mathrm{p}=0.01)$ compared to ESB patients. While ESB and NESB patients had similar rates in reporting any negative changes in behaviour and/or functional decline in activities of daily living, they had longer duration of symptoms (48 months on average) before receiving a formal diagnosis compared to ESB patients (30 months on average; $\mathrm{t}(46)=2.2$, $\mathrm{p}=0.03)$.

\subsection{Clinical Diagnostic Work-Up}

Both ESB and NESB patients had similar rates of referral for a thorough neuropsychology assessment (Table 1). While both groups had similar rates of structural neuroimaging with a CT and/or an MRI, the clinical diagnostic work-up on NESB patients (15 out of 19) relied 
more heavily on FDG PET compared to ESB patients (17 out of 35; Fisher exact test, $\mathrm{p}=0.04)$. Waiting times for FDG PET were the main cause of NESB patients delaying their feedback by 1.8 months on average compared to ESB patients $(\mathrm{t}(52)=2.1, \mathrm{p}=0.04)$.

\subsection{Dementia Subtype Diagnoses}

Thirty-two (59.3\%) were diagnosed with dementia, and of these 11 (34\%) were diagnosed with non-AD dementias (Table 2). Presentation rates of dementia as well as depression and/or anxiety without cognitive impairment in ESB and NESB patients were similar. However, a higher proportion of ESB patients (17 out of 35) compared to NESB patients (4 out of 19) were diagnosed with the $\mathrm{AD}$ dementia subtype alone (Fisher exact test, $\mathrm{p}$ $=0.08)$. NESB patients who presented with dementia (6 out of 10) were also more likely to be diagnosed with non-AD dementia compared to ESB patients (5 out of 22; Fisher exact test, $\mathrm{p}=0.06$ ).

\section{DISCUSSION}

\subsection{Presentation Patterns and Diagnostic Trends}

Baseline demographic findings are similar to previous reports [1,2]. NESB patients experienced symptoms 18 months longer on average before receiving a formal diagnosis, supporting a previous report of increased symptom severity at presentation [2]. It was reported in the same study that NESB patients were younger than ESB patients, which may be explained by a significantly smaller proportion of older elderly female NESB patients presenting.

The findings of a higher proportion of NESB patients with dementia presenting with non-AD dementia subtypes compared to ESB patients differs from LoGiudice et al. (2001). It is known that the unique cognitive and behavioural manifestations of non-AD dementias can often be discounted for psychiatric disorders clinically [10]. However the integration of FDG PET in the diagnostic work-up may have increased confidence in diagnosing non-AD dementia subtypes in our NESB cohort, which could account for the discrepancy in our results.

\subsection{Factors That May Impact on Patients' and Carers' Willingness to Attend a Memory Clinic}

Amnesia is the main cognitive deficit experienced initially in a typical AD sufferer. Our NESB patients were less likely to present with $\mathrm{AD}$ dementia subtype as the

Table 1. Patient characteristics.

\begin{tabular}{|c|c|c|c|c|c|c|c|}
\hline \multirow{2}{*}{ Patients } & \multicolumn{3}{|c|}{$\begin{array}{c}\text { English speaking background } \\
\text { (ESB) }\end{array}$} & \multicolumn{3}{|c|}{$\begin{array}{l}\text { Non-English speaking background } \\
\text { (NESB) }\end{array}$} & \multirow{2}{*}{$\begin{array}{l}\text { All } \\
\text { combined }\end{array}$} \\
\hline & Male & Female & Combined & Male & Female & Combined & \\
\hline Age & $74.7(15.1)$ & $74.9(11.2)$ & 74.8(12.9) & 74.1(10.9) & $67.0(11.6)$ & $71.2(11.5)$ & 73.5(12.5) \\
\hline$\leq 75$ years of age & 6 & 8 & 14 & 5 & 7 & 12 & 26 \\
\hline$>75$ years of age ${ }^{a}$ & 10 & 11 & 21 & 6 & 1 & 7 & 28 \\
\hline Living with spouse/partner & 13 & 8 & 21 & 8 & 5 & 13 & 34 \\
\hline Living alone $\mathrm{e}^{\mathrm{b}}$ & 3 & 6 & 9 & 1 & 0 & 1 & 10 \\
\hline Secondary education and above $e^{c}$ & 10 & 11 & 21 & 4 & 2 & 6 & 17 \\
\hline Mini Mental State Examination (MMSE) score ${ }^{d}$ & $24.1(3.4)$ & $22.2(4.3)$ & $23.1(4.0)$ & $18.6(5.5)$ & $21.4(5.0)$ & $19.8(5.4)$ & $21.9(4.8)$ \\
\hline Reported negative change in behavior or function & 10 & 14 & 24 & 9 & 6 & 15 & 39 \\
\hline Structural neuroimaging (MRI \pm CT/CT only) & $10 / 4$ & $14 / 3$ & $24 / 7$ & $7 / 3$ & $7 /$ & $14 / 3$ & $38 / 10$ \\
\hline Neuropsychology assessment & 9 & 14 & 23 & 10 & 6 & 16 & 39 \\
\hline FDG PET ${ }^{\mathrm{e}}$ & 9 & 8 & 17 & 9 & 6 & 15 & 32 \\
\hline Months from initial consultation to feedback ${ }^{f}$ & $2.4(2.3)$ & $5.0(2.8)$ & $3.8(2.8)$ & $5.7(2.8)$ & $5.5(4.0)$ & $5.6(3.2)$ & $4.5(3.1)$ \\
\hline Months from start of symptoms to feedback ${ }^{g}$ & 27(18) & $33(22)$ & $30(20)$ & $41(36)$ & 59(35) & $48(36)$ & $36(27)$ \\
\hline
\end{tabular}

${ }^{\mathrm{a} C o m p a r i n g}$ proportion of females of $>75$ years of age between ESB and NESB patients (Fisher exact test, $\mathrm{p}=0.04$ ). ${ }^{\mathrm{b}}$ Comparing ESB to NESB patients who reported living alone (Fisher exact test, $\mathrm{p}=0.08$ ). ${ }^{\mathrm{c}}$ Comparing ESB patients to NESB patients who had at least secondary school education (Fisher exact test, $\mathrm{p}$ $<0.001)$. ${ }^{\mathrm{d} C o m p a r i n g ~ M M S E ~ s c o r e s ~ b e t w e e n ~ E S B ~ a n d ~ N E S B ~ p a t i e n t s ~}(\mathrm{t}(51)=2.57, \mathrm{p}=0.01)$. ${ }^{\mathrm{e}}$ Comparing the use of FDG PET in the diagnostic work-up between ESB and NESB patients (Fisher exact test, $p=0.04$ ). ${ }^{\mathrm{f}}$ Comparing the duration between the initial consultation to receiving a formal diagnosis between ESB and NESB patients $(\mathrm{t}(52)=2.1, \mathrm{p}=0.04)$. ${ }^{\mathrm{g}}$ Comparing the duration between the onset of symptoms to receiving a formal diagnosis between ESB and NESB patients $(\mathrm{t}(46)=2.2, \mathrm{p}=0.03)$. 
Table 2. Diagnoses.

\begin{tabular}{|c|c|c|c|c|c|c|c|}
\hline \multirow{2}{*}{ Patients } & \multicolumn{3}{|c|}{ English speaking background (ESB) } & \multicolumn{3}{|c|}{ Non-English speaking background (NESB) } & \multirow{2}{*}{$\begin{array}{c}\text { Combined } \\
\text { total }\end{array}$} \\
\hline & Male & Female & Total & Male & Female & Total & \\
\hline Normal & 0 & 1 & 1 & 1 & 1 & 2 & 3 \\
\hline $\begin{array}{l}\text { Depression \&/or anxiety without } \\
\text { cognitive impairment }\end{array}$ & 4 & 3 & 7 & 1 & 2 & 3 & 10 \\
\hline Mild cognitive impairment & 1 & 0 & 1 & 3 & 0 & 3 & 4 \\
\hline Mixed AD and non-AD dementias ${ }^{b}$ & $1^{\mathrm{A}}$ & $4^{\mathrm{B}}$ & 5 & $3^{\mathrm{C}}$ & $1^{\mathrm{D}}$ & 4 & 9 \\
\hline Frontotemporal dementia (FTD) ${ }^{b}$ & 0 & 0 & 0 & 0 & 1 & 1 & 1 \\
\hline Vascular dementia (VD) ${ }^{b}$ & 0 & 0 & 0 & 1 & 0 & 1 & 1 \\
\hline Other & 2 & 2 & 4 & 0 & 1 & 1 & 5 \\
\hline
\end{tabular}

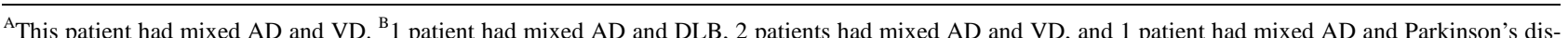
ease dementia. ${ }^{\mathrm{C}} 2$ patients had mixed $\mathrm{AD}$ and FTD, and 1 patient had mixed AD and DLB. ${ }^{\mathrm{D}}$ This patient had mixed AD and DLB. ${ }^{\mathrm{a}}$ Comparing rates of AD (without non-AD dementia) between all presenting ESB and NESB patients (Fisher exact test, $\mathrm{p}=0.08$ ). ${ }^{\mathbf{b}}$ Comparing total rates of non-AD dementias between ESB and NESB patients who present with dementia (Fisher exact test, $\mathrm{p}=0.06$ ).

sole cognitive diagnosis, perhaps because they and their carers had more tolerance towards higher degrees of amnesia before help was sought. This may be because of differences in available family supports and in the cultural expectations of caregiving placed on family members (as suggested by NESB patients being more likely to live with someone), relative isolation due to cultural and language barriers (as they perform worse on cognitive testing which are culturally and language biased), lack of education about dementia and available services (as suggested by them being significantly less educated), and social stigma in seeking help (as suggested by an under representation of older elderly females). It has also been shown that there is preservation or enhancement of social-emotional function in early AD [11], which may be a barrier to presentation paradoxically.

In contrast to $\mathrm{AD}$, other cognitive domains to memory may be affected first in non-AD subtypes. For example, the salient clinical characteristic of behavioural variant FTD is a profound alteration in character and social conduct, occurring in the context of relative preservation of instrumental functions of perception, spatial skills, praxis and memory [12]. Unlike early AD, behavioural variant FTD has an inverse pattern to the enhancement of socialemotional function [11]. Also in DLB, sufferers often experience movement disorders, fluctuations in cognitive performance and level of consciousness, psychosis, and major depressive episodes, yet there is often a relative preservation of short term memory [13]. Hence deficits in non-memory cognitive domains which are associated with non-AD dementia tend to have greater impact on patients' functional activities of daily living and levels of carer burden. Our data supports the notion that push factors (e.g. carers' stress) rather than pull factors (e.g. patient desiring a thorough checkup to see if their perceived cognitive decline is abnormal) contributed more to NESB patients attending [2].

\subsection{Clinical and Social Implications}

There is consensus that an earlier diagnosis of dementia could improve outcome. A modest improvement is cost effective, and may delay admission into long term care, which is the main driver of the direct costs of dementia [14,15]. Unfortunately, memory clinic services assess only a very small proportion of people with dementia in any country or region $[1,16]$. Increasing the availability of FDG PET to other appropriate specialists may provide a more resource-efficient approach.

It is important to diagnose dementia accurately as treatment approaches are different. For example, neuroleptic sensitivity is more common with DLB which treating clinicians must be mindful of when managing psychosis [13]. Furthermore while acetylcholinesterase inhibitors are effective in AD and DLB [17], they may exacerbate symptoms in FTD [18]. Hence the previously reported increase in psychiatric disorders in presenting NESB patients [2] which could lead to under-diagnosis of non-AD dementias warrants further study.

\subsection{Use of FDG PET in the Diagnostic Work-Up of Dementia}

The diagnoses of dementia subtypes made in our cohort were consistent with standard clinical criteria. However applying the standard clinical criteria for diagnosis is open to a degree of subjectivity [19]. Hence centres of excellence are increasingly moving towards the use of biomarkers like FDG PET to improve accuracy in the diagnostic work-up [20]. FDG PET is an adjunct tool where an abnormal scan raising the possibility of neurodegenerative pathology in vivo is not itself a diagnosis of dementia [3]. The rate of diagnosing dementia as a whole 
in the AMS (59.3\%) has not increased compared to other memory clinics $(>60 \%)$, despite our extensive use of FDG PET. However we may be diagnosing more mixed $\mathrm{AD}$ and non-AD dementias (17\%).

\subsection{Limitations}

The limitations of this study are the single centre setting, the small numbers, the heterogeneous NESB patient group, and it was not powered to detect for increased rate of psychiatric disorders amongst NESB patients. The AMS covers a different catchment area to that in previous reports, and conclusions drawn from this study may not apply to clinics serving other populations. Nonetheless there is considerable similarity between patients from our catchment area and that in at least one other study [2], which is reflected in our similar baseline demographic findings. Finally a high quality diagnostic work-up that involves the use of FDG PET may improve sensitivity and specificity of diagnoses, but a definitive diagnosis of any neurodegenerative subtype is generally made at post mortem.

\section{CONCLUSION}

ESB and NESB patients may have different responses towards $\mathrm{AD}$ and non-AD dementias, resulting in the propensity for NESBs to present with non-AD dementia. Memory service provision to older elderly NESB females may be under utilised. Our findings support an integrative approach with the use of biomarkers to the diagnostic work-up, particularly for NESB patients who may have language barriers. Larger studies are required to validate these preliminary findings.

\section{ACKNOWLEDGEMENTS}

K. Ong designed the study, collected and analyzed the data, and wrote the paper. M. Woodward supervised the study, assisted with writing, and made critical revisions to the paper. The authors would like to thank Ms Kaye Milburn and Ms Paula Jack for their help in locating patient folders and supporting this study.

\section{REFERENCES}

[1] Woodward, M.C. and Woodward, E. (2009) A national survey of memory clinics in Australia. International Psychogeriatrics, 21, 696-702. doi:10.1017/S1041610209009156

[2] LoGiudice, D., Hassett, A., Cook, R., et al. (2001) Equity of access to a memory clinic in Melbourne? Non-English speaking background attenders are more severely demented and have increased rates of psychiatric disorders. International Journal of Geriatric Psychiatry, 16, 327-334. doi:10.1002/gps.346

[3] Bohnen, N.I., Djang, D.S., Herholz, K., et al. (2012) Ef- fectiveness and safety of 18F-FDG PET in the evaluation of dementia: A review of the recent literature. Journal of Nuclear Medicine, 53, 59-71. doi:10.2967/jnumed.111.096578

[4] Shaffer, J.L., Petrella, J.R., Sheldon, F.C., et al. (2013) Predicting cognitive decline in subjects at risk for Alzheimer disease by using combined cerebrospinal fluid, MR imaging, and PET biomarkers. Radiology, 266, 583-591. doi:10.1148/radiol.12120010

[5] Roth, M., Tym, E., Mountjoy, C.Q., et al. (1986) CAMDEX: A standardised instrument for the diagnosis for mental disorder in the elderly with a special reference to early detection of dementia. British Journal of Psychiatry, 149, 698-709. doi:10.1192/bjp.149.6.698

[6] Mioshi, E., Dawson, K., Mitchell, J., et al. (2006) The Addenbrooke's Cognitive Examination Revised (ACE-R): A brief cognitive test battery for dementia screening. International Journal of Geriatric Psychiatry, 21, 10781085. doi:10.1002/gps.1610

[7] Koenig, H.G., Meador, K.G., Cohen, H.J., et al. (1988) Self-rated depression scales and screening for major depression in the older hospitalized patient with medical illness. Journal of the American Geriatrics Society, 36, 699-706.

[8] Folstein, M.F., Folstein, S.E. and McHugh, P.R. (1975) "Mini-mental state". A practical method for grading the cognitive state of patients for the clinician. Journal of Psychiatric Research, 12, 189-198. doi:10.1016/0022-3956(75)90026-6

[9] Petersen, R.C. (2004) Mild cognitive impairment as a diagnostic entity. Journal of Internal Medicine, 256, 183194. doi:10.1111/j.1365-2796.2004.01388.x

[10] Hou, C.E., Carlin, D. and Miller, B.L. (2004) Non-Alzheimer's disease dementias: Anatomic, clinical, and molecular correlates. Canadian Journal of Psychiatry, 49, 164-171.

[11] Zhou, J., Greicius, M.D., Gennatas, E.D., et al. (2010) Divergent network connectivity changes in behavioural variant frontotemporal dementia and Alzheimer's disease. Brain, 133, 1352-1367. doi:10.1093/brain/awq075

[12] Snowden, J.S., Neary, D. and Mann, D.M.A. (2002) Frontotemporal dementia. British Journal of Psychiatry, 180, 140-143. doi:10.1192/bjp.180.2.140

[13] McKeith, I.G. (2002) Dementia with Lewy Bodies. British Journal of Psychiatry, 180, 144-147. doi:10.1192/bjp.180.2.144

[14] Banerjee, S. and Wittenberg, R. (2009) Clinical and cost effectiveness of services for early diagnosis and intervention in dementia. International Journal of Geriatric Psychiatry, 24, 48-54. doi:10.1002/gps.2191

[15] Prince, M., Bryce, R. and Ferri, C. (2011) Alzheimer's Disease International World Alzheimer Report 2011. The benefits of early diagnosis and intervention. Alzheimer's Disease International.

[16] Moise, P., Schwarzinger, M. and Um, M.-Y. (2004) Demetia Expert's Group. Dementia Care in 9 OECD Countries: A comparative analysis. OECD Health Working Papers, 13. 
[17] Seltzer, B. (2007) Donepezil: An update. Expert Opinion on Pharmacotherapy, 8, 1011-1023. doi:10.1517/14656566.8.7.1011

[18] Mendez, M.F., Shapira, J.S., McMurtray, A., et al. (2001) Preliminary findings: Behavioral worsening on donepezil in patients with frontotemporal dementia. American Journal of Geriatric Psychiatry, 15, 84-87. doi:10.1097/01.JGP.0000231744.69631.33

[19] Lopez, O.L., Litvan, I., Catt, K.E., et al. (1999) Accuracy of four clinical diagnostic criteria for the diagnosis of neurodegenerative dementias. Neurology, 53, 1292. doi:10.1212/WNL.53.6.1292

[20] Albert, M.S., DeKosky, S.T., Dickson, D., et al. (2011) The diagnosis of mild cognitive impairment due to Alzheimer's disease: Recommendations from the National Institute on Aging-Alzheimer's Association workgroups on diagnostic guidelines for Alzheimer's disease. Alzheimers \& Dementia, 7, 270-279.

doi:10.1016/j.jalz.2011.03.008

\section{Supplementary Table 1}

\begin{tabular}{cc}
\hline NESB country of origin & $\mathbf{N}(\%)$ \\
\hline Italy & $4(21)$ \\
Malta & $4(21)$ \\
Greece & $2(11)$ \\
Turkey & $2(11)$ \\
Chile & $1(5)$ \\
Argentina & $1(5)$ \\
Macedonia & $1(5)$ \\
China & $1(5)$ \\
Switzerland & $1(5)$ \\
Lebanon & $1(5)$ \\
Unknown & $1(5)$ \\
\hline
\end{tabular}

